\title{
Response to the article by Dietz et al. entitled "Recommendations for prioritization, treatment, and triage of breast cancer patients during the COVID-19 pandemic. The COVID-19 pandemic breast cancer consortium"
}

\author{
Peter Beitsch $^{1}$ (1) $\cdot$ Pat Whitworth $^{2} \cdot$ Rakesh Patel $^{3}$
}

Received: 10 May 2020 / Accepted: 14 May 2020 / Published online: 26 May 2020

(c) Springer Science+Business Media, LLC, part of Springer Nature 2020

To the editor,

Dietz et al. should be commended for the coordinated effort undertaken to convene the first ever multi-society [COVID19] Pandemic Breast Cancer Consortium. This framework can be deployed for future rapid-response situations in which adjustments to standard breast cancer treatment guidance are required.

Although we agree with most of the guidance, one topic that merits further consideration. In the Medical Oncology section for ER+, HER2- invasive breast cancer, the authors point out that some of these cancers may have surgical intervention delayed with the administration of neoadjuvant endocrine therapy based upon clinical parameters or genomic assay results. Unfortunately, the language in this section elevates a particular commercial test by including the following statement "especially Recurrence Score, which may be sent on a core biopsy." Additionally, the only citations referenced for "genomic assays" or "luminal A signatures" pertain to the same Recurrence Score test. Based on these current references, the reader might incorrectly conclude the following two points: (1) only the 21-gene Recurrence Score has supportive data for use on core biopsy and

This comment refers to the article available online at https://doi. org/10.1007/s10549-020-05644-z.

Peter Beitsch

beitsch@aol.com

Pat Whitworth

patwhitworth@gmail.com

Rakesh Patel

iupatel@yahoo.com

1 Dallas Breast Center, Dallas, TX, USA

2 Nashville Breast Center, Nashville, TN, USA

3 Good Samaritan Breast Care Center, Los Gatos, CA, USA
(2) a "Luminal A" result can be derived from the 21-gene Recurrence Score. For the first point, we firmly believe that it was not the intention of the consortium to recommend a preference for one test over another and believe that the cited references should be updated to reflect a more comprehensive list of available genomic tests. On the second point, the 21-gene Recurrence Score cannot provide molecular subtyping information, so any implication that it has this ability is incorrect. The only commercially available test in the US that can report molecular subtyping to distinguish Luminal patients from other subtypes is the 80-gene test (Agendia, BluePrint). While the 50-gene assay (Prosigna) is also capable of molecular subtyping, that functionality is not available in the US market.

Furthermore, the statement regarding the likelihood of benefit from neoadjuvant chemotherapy (NCT) in patients with a "low-risk" genomic test result does not reference trials or studies where NCT was actually administered. We were the primary investigators on a large prospective neoadjuvant breast cancer treatment trial exploring real-world utility of genomic testing on core biopsy with the 70-gene and 80-gene assays (NBRST, clinicaltrials.gov identifier: NCT01479101). We feel that this important data can elucidate molecular subtype-driven response which would be helpful to include in this consortium guidance as a means to identify those patients most likely to achieve pCR $[1,2]$. Importantly, observations from the NBRST study demonstrated that approximately 1 out of 4 patients are reclassified from their clinical subtype (based on IHC/FISH) to a different functional molecular subtype, which aligns more precisely with their observed response to NCT $[1,2]$. These clinically ER+, molecularly Basal patients have a 34\% pCR rate with NCT similar to pathologically "triple-negative" patients (TNBC) where delays in systemic treatment or surgery can negatively impact outcomes. Consistent with these 
findings, the prospective I-SPY 2 trial (ClinicalTrials.gov Identifier: NCT01042379) has confirmed the presence and predictive value of this ER+ Basal-type profile, which was identified in almost $30 \%$ of clinically ER+, HER $2-$ patients in the trial.

In comparison, there are currently no such data that support the utility of the 21-gene Recurrence Score's ability to identify these molecular profiles. Further, one of the more recent retrospective analyses only indicated a $9.6 \%$ pCR rate with high-risk $\mathrm{RS}(\mathrm{RS} \geq 31$ ), which is no better than traditional clinical classification with IHC [3].

This current pandemic situation has altered the norm for treating breast cancer and provided a rare opportunity for all of us to re-evaluate our clinical habits in the interest of improving patient care. Whether it is revisiting the mammographic screening interval or moving to the use of genomic testing from the core biopsy to help guide pre-operative treatment planning (e.g., surgery vs. NCT), a fresh look at our current protocols is both a necessity and an opportunity.

Best Regards,

Peter Beitsch, MD—Past President, American Society of Breast Surgeons
Pat Whitworth, MD—Past Chairman of the Board, American Society of Breast Surgeons

Rakesh Patel, MD—Past President, American Brachytherapy Society

\section{References}

1. Whitworth P, Stork-Sloots L, de Snoo FA (2014) Chemosensitivity predicted by BluePrint 80 -gene functional subtype and MammaPrint in the Prospective Neoadjuvant Breast Registry Symphony Trial (NBRST). Ann Surg Oncol 21(10):3261-3267

2. Beitsch P, Whitworth P, Baron P (2017) Pertuzumab/trastuzumab/ CT versus trastuzumab/CT therapy for HER2+ breast cancer: results from the prospective Neoadjuvant Breast Registry Symphony Trial (NBRST). Ann Surg Oncol 24(9):2539-2546

3. Pease AM, Riba LA, Gruner RA (2019) Oncotype DX recurrence score as a predictor of response to neoadjuvant chemotherapy. Ann Surg Oncol 26(2):366-371

Publisher's Note Springer Nature remains neutral with regard to jurisdictional claims in published maps and institutional affiliations. 